\title{
Composición taxonómica y abundancia espacio-temporal de la ictiofauna del Río Presidio, Sinaloa, México
}

\author{
Sergio Sánchez-Gonzáles ${ }^{1}$, Gorgonio Ruiz-Campos ${ }^{2, *}$, Alejandro Herrrera-Flores ${ }^{1}$,
}

María de Lourdes Lozano-Vilano ${ }^{3}$, Adrián F. González-Acosta ${ }^{4}$, Héctor Manuel Inzunza-Beltrán ${ }^{1}$

1. Facultad de Biología, Universidad Autónoma de Sinaloa, Av. Universitarios y de las Américas s/n, Col. Universitarios (Ciudad Universitaria), Culiacán, Sinaloa, 80010, México; ssanchez@uas.edu.mx

2. Facultad de Ciencias, Universidad Autónoma de Baja California, carretera Transpeninsular Ensenada-Tijuana No. 3917, colonia Playitas, Ensenada, Baja California, 22860, México; gruiz@uabc.edu.mx

3. Facultad de Ciencias Biológicas, Universidad Autónoma de Nuevo León, San Nicolás de los Garza, Nuevo León, 66455, México; maria.lozanovl@uanl.edu.mx

4. Instituto Politécnico Nacional-Centro Interdisciplinario de Ciencias Marinas, Av. Instituto Politécnico Nacional s/n, La Paz, Baja California Sur, 23096, México; aacosta@ipn.mx

* Correspondencia

Recibido 14-X-2017. Corregido 08-III-2018. Aceptado 06-IV-2018.

\begin{abstract}
Taxonomic composition and spatio-temporal abundance of the ichthyofauna in Presidio River, Sinaloa, Mexico. The Rio Presidio in the southern Sinaloa, Mexico belongs to Nearctic-Neotropical zoogeographical transition zone, where its fish fauna could manifest changes in the abundance and distribution of the species during rainy and dry periods. The main objective of this study was to describe the taxonomic composition and spatial and temporal abundance of the fish fauna from the lower and middle Rio Presidio, by means of bimonthly samplings from April 2008 to February 2009. Forty fish species (39 native and 1 exotic) belonging to 32 genera and 19 families were registered. Two families (Gerreidae and Poeciliidae) contained the highest numbers of species (five and four, respectively). Six species (Lile stolifera, Oreochromis aureus, Awaous banana, Anchoa analis, Gobiomorus maculatus and Atherinella crystallina) in this order of importance contributed with almost $49 \%$ of the total fish abundance. Species richness and salinity showed a direct relationship and an inverse relationship with the altitude. Based on the coefficient of similarity of species among sites two fish assemblages were identified. The first assemblage formed by species from marine derivation (peripheral) occurring in the lower part of the river, and the second assemblage by secondary freshwater species in the middle part. The higher fish species similarity observed in August and September was under the influence of the highest flows of the rainy period. Twenty-seven 27 species showed affinity to the Tropical Eastern Pacific Region, six species to the Neotropical region and two to the Nearctic region (Dorosoma smithi and Ictalurus cf. pricei ); one species has an Amphiatlantic distribution (Mugil curema), another is circumtropical (Mugil cephalus), two species have Amphiamerican distribution (A. monticola and G. cinereus), and one is exotic (O. aureus). Rev. Biol. Trop. 66(2): 848-862. Epub 2018 June 01.
\end{abstract}

Key words: fish fauna; distribution; abundance; zoogeography; ecogeography.

Una de las regiones del noroeste de México que cuenta con el mayor número de cuencas hidrológicas es el Estado de Sinaloa, con un total de once ríos de tercer orden que drenan hacia el Golfo de California y el océano Pacífico (Tamayo \& West, 1964; INEGI, 1995). En ese conjunto de corrientes epicontinentales se tienen registradas 71 especies de peces (Miller et al., 2005), de las cuales 15 son reportadas como endémicas del noroeste de México (Espinosa-Pérez et al., 1993; Miller et al., 2005). En esta misma área geográfica, los límites de las regiones biogeográficas Neártica y Neotropical quedan debidamente expresados a través del componente ictiológico dentro de cada cuenca, dominando formas neárticas en la parte 
alta, formas neotropicales en la parte media, y formas de derivación marina en la parte baja (Miller et al., 2005).

El Río Presidio, localizado en el sur del Estado de Sinaloa, es uno de los sistemas hidrológicos cuyas aguas, en su parte media, han sido recientemente represadas (abril de 2009) por la construcción de la presa Picachos, lo que posiblemente tendrá efectos sobre la distribución y abundancia de la ictiofauna nativa y otra biota asociada, debido a los cambios en los niveles de flujo y la interrupción de los eventos de migración reproductiva de especies diádromas (e.g., Agonostomus monticola Bancroft, 1834).

Jordan (1895) en su obra sobre los peces de Sinaloa, reportó 18 especies para las localidades de Villa Unión y Presidio en el Río Presidio, aportando la descripción de tres nuevas especies: Poeciliopsis presidionis (Jordan \& Culver, 1895), Pseudophallus starksii (Jordan \& Culver, 1895) y Atherinella crystallina (Jordan \& Culver, 1895).

Aunque existen registros puntuales de diferentes especies de peces presentes en la cuenca del Río Presidio de Sinaloa (e.g., Jordan, 1895; Álvarez del Villar, 1970; Castro-Aguirre et al., 1976; Castro-Aguirre et al., 1999; Miller et al., 2005), ninguno ha evaluado en un contexto espacial y temporal, la composición íctica de este ecosistema.

Considerando la escasa información que existe sobre la ictiofauna dulceacuícola del Estado de Sinaloa y, en especial del Río Presidio, se realizó una evaluación de la composición y abundancia espacio-temporal de la fauna íctica en la parte media y baja de la cuenca, previo a la construcción de la presa Picachos que finalizó en julio de 2009.

\section{MATERIALES Y MÉTODOS}

Área de estudio: El presente estudio se realizó en el periodo entre abril 2008 y febrero 2009, en las cuencas media y baja del Río Presidio que se localiza en el Estado de Sinaloa, México $\left(23^{\circ} 35^{\prime}-23^{\circ} 04^{\prime} \mathrm{N}\right.$ y $106^{\circ} 18^{\prime}-106^{\circ} 05^{\prime}$ $\mathrm{W})$. Este río, de $167 \mathrm{~km}$ de longitud, nace en la Sierra Madre Occidental en el Estado de Durango y desemboca en el estero El Ostial, cerca de Villa Unión, Municipio de Mazatlán. $\mathrm{Su}$ área de cuenca es de $5450 \mathrm{~km}^{2}$ y su descarga media anual es de 1779 millones de $\mathrm{m}^{3}$ (Tamayo \& West, 1964; De la Lanza \& GarcíaCalderón, 1991).

El clima de la región donde se ubica la cuenca del Río Presidio es cálido sub-húmedo, con una temperatura media anual de $24{ }^{\circ} \mathrm{C}$ y la precipitación varía de los 800 a $1200 \mathrm{~mm}$ (García, 2004). El suelo es de origen aluvial con un alto porcentaje de arena y conglomerados de reciente depósito en las márgenes del río. La cubierta vegetal dominante es selva baja caducifolia, la cual se extiende desde la parte media de la sierra donde existen bosques de encino y asociaciones de pino-encino, hasta la costa donde se presentan importantes áreas de manglar y vegetación halófila (Vega-Aviña, 2002; Sicairos-Avitia et al., 2003).

\section{Muestreos ictiológicos y de parámetros} físico-químicos: Diez sitios entre la parte media y baja del Río Presidio fueron seleccionados para este estudio (Fig. 1), en los cuales se realizaron seis muestreos de peces durante el periodo de abril 2008 a febrero 2009 (19-20 abril, 17-18 mayo, 9-10 agosto, 13-14 septiembre, 7-8 noviembre, y 11-12 febrero). Dichos sitios fueron clasificados en dos secciones de acuerdo con su altitud en metros sobre nivel medio del mar (msnm), como: 1) la parte media representada por las localidades de Los Humayes (LH,150 msnm), Los Copales (LC, $128 \mathrm{msnm}$ ), Las Iguanas (LI, $102 \mathrm{msnm}$ ), La Cortina (LCO, $83 \mathrm{msnm}$ ), El Recodo (ER, $56 \mathrm{msnm}$ ) y la Derivadora Siqueros (DS, 33 msnm); y 2) la parte baja representada por El Walamo (EW, 4 msnm), Barrón-Ostial (BO, 2 $\mathrm{msnm}$ ), El Botadero (EB, $5 \mathrm{msnm}$ ) y Las Garzas (LG, $3 \mathrm{msnm}$ ). Los peces fueron capturados con atarrayas de luz de malla de $1 / 4$ " (altura 2.5 m), $1 / 2$ " (altura $2.5 \mathrm{~m}$ ) y $2 \frac{1}{2}$ " (altura $3 \mathrm{~m}$ ), un chinchorro de arrastre (4 x $2 \mathrm{~m}$ y luz de malla de ${ }_{1 / 8} "$ ) y una red tipo Surber (bolsa de 100 $\mathrm{cm}$ de longitud y luz de malla de 500 micras, conectada a un marco de acero inoxidable de 


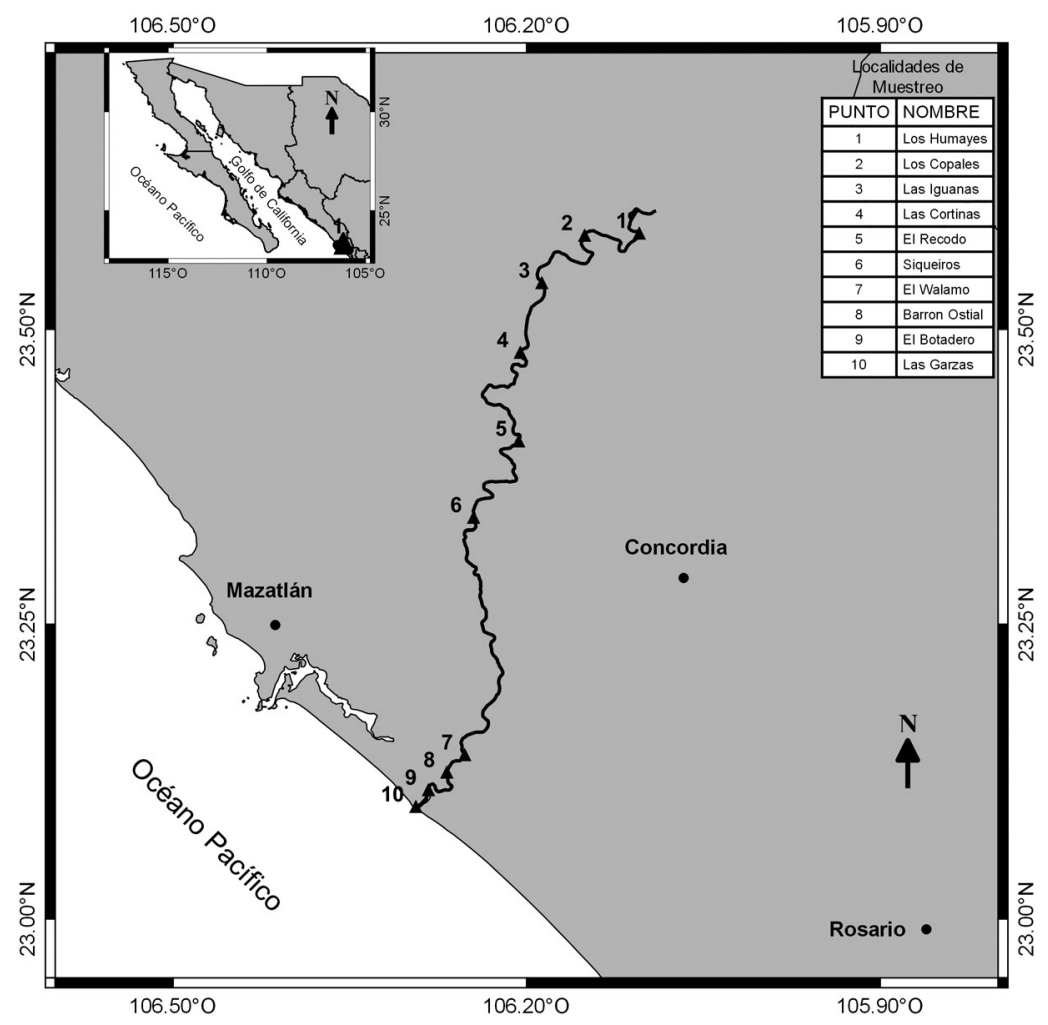

Fig. 1. Localidades de muestreo de peces en el Río Presidio, Sinaloa, México, de abril 2008 a febrero 2009.

Fig. 1. Fish sampling sites along the Rio Presidio, Mazatlán, Sinaloa, Mexico, during April 2008 to February 2009.

$60 \times 40 \mathrm{~cm}$ ) para muestreo en hábitats con fondos de canto rodado. El esfuerzo de recolecta en cada sitio y fecha de muestreo fue de 20 a 25 lances con atarraya, así como 40 a 45 minutos de muestreo con chinchorro de arrastre y de 40 a 45 minutos con la red de fondo (Surber).

Los organismos capturados fueron fijados en formaldehído al $10 \%$ y posteriormente preservados en alcohol isopropílico al $50 \%$. La determinación taxonómica de los especímenes fue con ayuda de las claves de identificación de Fischer et al. (1995), Castro-Aguirre et al. (1999) y Miller et al. (2005); mientras que la identificación de Awaous banana fue basada en Watson (1996) y Diapterus brevirostris en González-Acosta et al. (2007). El arreglo sistemático y nomenclatura de las especies sigue lo establecido por Page et al. (2013).
El material ictiológico fue depositado en la Colección Ictiológica de la Universidad Autónoma de Baja California (UABC) y en la Colección de Peces del Laboratorio de Conservación de la Fauna Silvestre de la Facultad de Biología de la Universidad Autónoma de Sinaloa (UAS). En el cuadro 2 se ofrece el número de catálogo de especímenes selectos de cada especie.

La clasificación ecogeográfica se elaboró con base en los criterios establecidos por Myers (1951) modificada por Castro-Aguirre et al. (1999). Mientras que las afinidades zoogeográficas de las especies del componente marino fueron basadas en Briggs (1974), y aquéllas de las especies de estirpe continental en Darlington (1963) y Miller et al. (2005).

Durante los muestreos de peces se midieron las siguientes variables físico-químicas del 
agua con ayuda de un equipo multianalizador Hydrolab Scout DS5: temperatura $\left({ }^{\circ} \mathrm{C}\right)$, potencial de hidrógeno, oxígeno disuelto (mg/l), salinidad (\%o) y turbidez (NTU).

La similitud de especies espacio-temporal de la comunidad íctica se realizó el coeficiente de similitud de Jaccard, calculado mediante la expresión: $\mathrm{CCJ}=\mathrm{c} /[\mathrm{a}+\mathrm{b}-\mathrm{c}]$, donde "a" $\mathrm{y}$ "b" son el número de especies en las localidades 1 y 2 , respectivamente, y "c" el número de especies compartidas entre ambas localidades. Para este análisis se aplicó el algoritmo de agrupamiento de pares promediados ponderados y la métrica de distancia de Chebychev, con ayuda del paquete Statistica 6.0 (StatSoft, Inc., Tulsa, OK, 2002).

Un análisis de componentes principales fue procesado para determinar las variables ambientales y bióticas que mejor explican la variación en la composición y riqueza de especies de la parte media y baja del río de estudio, considerando la altitud la como variable suplementaria y las localidades como la variable de agrupamiento.

\section{RESULTADOS}

Parámetros fissico-químicos: Los valores promedio, mínimos y máximos de los parámetros físico-químicos del agua medidos en las diferentes localidades del muestreo ictiológico se presentan en el cuadro 1. La salinidad presentó pequeñas variaciones desde la parte alta $(0.11 \%=$ Los Humayes $)$ hacía la parte baja de la cuenca $(0.14 \%$ = Barrón-Ostial $)$, con incrementos significativos más río abajo en las localidades cercanas a la planicie costera (El Botadero, 31.4 \%; y Las Garzas, $32.7 \%$ ), mismas que se caracterizan por la prevalecen condiciones marinas de tipo oligohalino y eurihalino.

\section{Composición y diversidad espacio-tem-} poral: Se capturó un total de 4101 organismos que corresponden a 40 especies, 32 géneros y 19 familias de teleósteos (Cuadro 2). El número de especies fue mayor para las familias Gerreidae (5) y Poeciliidae (4), mientras otras familias como Mugilidae, Lutjanidae, Eleotridae y Gobiidae, presentaron tres especies cada una.

La mayor riqueza de especies se registró en las localidades de la parte baja del río como El Botadero y Las Garzas con 31 y 21 especies, respectivamente (Cuadro 3). En las localidades de la parte media del río la riqueza específica osciló entre 9 (Los Humayes) y 15 (Derivadora Siqueros). A nivel temporal, la mayor riqueza de especies se presentó en los meses de secas,

\section{CUADRO 1}

Promedio e intervalo de parámetros físico-químicos del agua determinados por sitio de muestreo en la cuenca media y baja del Río Presidio, Mazatlán, Sinaloa, México, abril 2008-febrero 2009

TABLE 1

Average values and range of physicochemical parameters of water registered per sampling site in the middle and lower basin of the Rio Presidio, Mazatlán, Mexico, during April 2008 to February 2009

\begin{tabular}{lccccc}
\multicolumn{1}{c}{ Localidad } & Oxígeno disuelto $(\mathrm{mg} / \mathrm{l})$ & $\mathrm{pH}$ & Temperatura ${ }^{\circ} \mathrm{C}$ & Salinidad (\%o) & Turbidez (NTU) \\
Los Humayes & $7.6(3.4-9.7)$ & $8.5(7.7-8.9)$ & $24.3(20.8-28.4)$ & $0.11(0.11-0.13)$ & $1.7(0.8-2.7)$ \\
Los Copales & $8.5(5.5-10.0)$ & $8.8(8.4-9.3)$ & $26.3(23.0-29.8)$ & $0.12(0.10-0.13)$ & $1.8(1.0-2.8)$ \\
Las Iguanas & $8.4(5.0-10.4)$ & $8.7(8.3-9.2)$ & $26.3(22.8-31.0)$ & $0.12(0.12-0.13)$ & $1.7(0.7-3.0)$ \\
La Cortina & $6.2(4.4-6.9)$ & $8.1(8.0-8.4)$ & $26.842(23.0-32.7)$ & $0.13(0.12-0.13)$ & $7.3(4.2-12.5)$ \\
El Recodo & $8.7(4.6-12.2)$ & $8.7(8.3-9.1)$ & $26.7(24.1-29.9)$ & $0.13(0.10-0.13)$ & $6.6(2.1-10.7)$ \\
Siqueros & $6.8(4.9-9.1)$ & $8.2(7.9-8.3)$ & $23.6(21.8-25.5)$ & $0.12(0.10-0.13)$ & $11.1(5.7-17.0)$ \\
El Walamo & $3.7(3.4-4.1)$ & $8.4(8.2-8.6)$ & $26.6(26.4-26.7)$ & $0.13(0.13-0.14)$ & $17.2(12.2-22.1)$ \\
Barrón-Ostial & $5.9(4.6-7.1)$ & $8.9(8.4-9.2)$ & $26.5(25.3-27.5)$ & $0.14(0.13-0.16)$ & $26.6(23.7-30.9)$ \\
El Botadero & $7.2(4.4-9.5)$ & $7.8(7.7-8.0)$ & $28.2(25.7-29.7)$ & $31.4(23.4-37.3)$ & $23.0(21.1-34.1)$ \\
Las Garzas & $3.7(2.6-5.1)$ & $7.5(7.4-7.7)$ & $26.0(24.0-27.9)$ & $32.7(23.3-39.3)$ & $14.4(8.2-20.6)$ \\
\hline
\end{tabular}


CUADRO 2

Clasificación taxonómica, derivación ecológica, afinidad biogeográfica y registro curatorial de las especies ícticas recolectadas en el Río Presidio, Mazatlán, Sinaloa; abril 2008-febrero 2009

TABLE 2

Taxonomical classification, ecological derivation, biogeographic affinity and curatorial record of fish species collected in the Rio Presidio, Mazatlán, Sinaloa, during April 2008 to February 2009

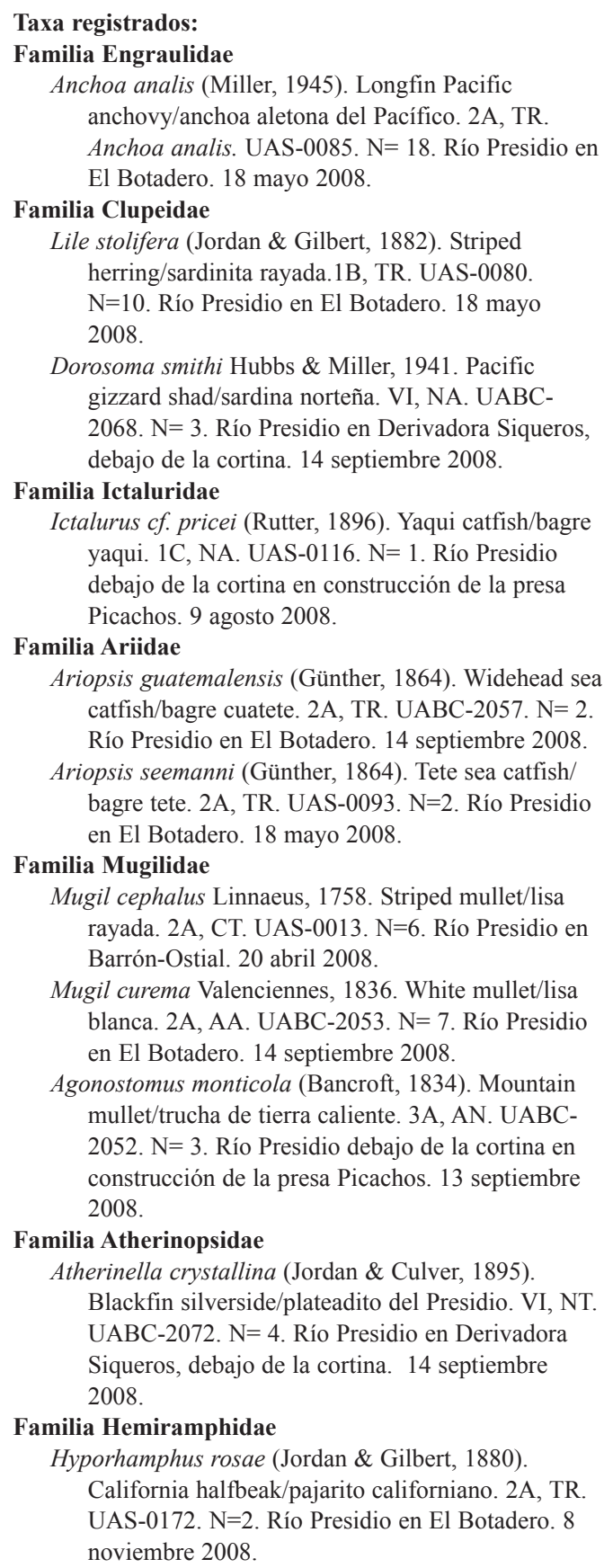

\section{Familia Poeciliidae}

Poecilia butleri Jordan, 1889. Pacific molly/topote del Pacífico. 2C, NT. UAS-00158. N=16. Río Presidio en la confluencia del Arroyo Copales. 7 noviembre 2008.

Poeciliopsis latidens (Garman, 1895). Lowland livebearer/guatopote del Fuerte. 2C, NT. UAS0156. $\mathrm{N}=2$. Río Presidio en la confluencia del Arroyo Copales. 7 noviembre 2008.

Poeciliopsis presidionis (Jordan \& Culver, 1895). Sinaloa livebearer/guatopote de Sinaloa. 2C, NT. UABC-2074. N=1. Río Presidio en El Recodo. 14 septiembre 2008.

Poeciliopsis prolifica Miller, 1960. Blackstripe livebearer/guatopote culiche. 2C, NT. UAS0008. N=4. Río Presidio debajo de la cortina en construcción de la presa Picachos. 19 abril 2008.

\section{Familia Centropomidae}

Centropomus nigrescens Günther, 1864. Black snook/ robalo negro. 2A, TR. UAS 0069. $\mathrm{N}=1$. Río Presidio en la Derivadora Siqueros. 18 mayo 2008.

Centropomus robalito Jordan \& Gilbert, 1882. Yellowfin snook/robalo aleta amarilla. 2A, TR. UAS-0092. N=1. Río Presidio en El Botadero. 18 mayo 2008.

Familia Carangidae

Caranx vinctus Jordan \& Gilbert, 1882. Cocinero/ cocinero. 2B, TR. UAS-0141. N=1. Río Presidio en El Botadero. 10 agosto 2008.

Selene brevoorti (Gill, 1863). Mexican lookdown/ jorobado mexicano. 2B, TR. UAS-0019. N=1. Río Presidio en El Botadero. 20 abril 2008.

Familia Lutjanidae

Lutjanus argentiventris (Peters, 1869). Amarillo snapper/pargo amarillo. 2B, TR. UABC-2055. $\mathrm{N}=2$. Río Presidio en El Botadero. 14 septiembre 2008.

Lutjanus colorado Jordan \& Gilbert, 1882. Colorado snapper/pargo colorado. 2B, TR. UAS-0086. N=1. Río Presidio en El Botadero. 18 mayo 2008.

Lutjanus novemfasciatus Gill, 1862. Pacific dog snapper/pargo prieto. 2A, TR. UAS-0089. N=1. Río Presidio en El Botadero. 18 mayo 2008.

\section{Familia Gerreidae}

Diapterus brevirostris (Sauvage, 1879). Yellowfin mojarra/mojarra aletas amarillas. 2A, TR. UAS0096. $\mathrm{N}=6$. Río Presidio en El Botadero. 18 mayo 2008. 
Eucinostomus currani Zahuranec, 1980. Pacific flagfin mojarra/mojarra tricolor. 2A, TR. UAS-0021. N=1. Río Presidio en El Botadero. 18 mayo 2008.

Eucinostomus entomelas Zahuranec, 1980. Darkspot mojarra/mojarra mancha negra. 2A, TR. UAS0083. N=3. Río Presidio en El Botadero. 18 mayo 2008.

Eugerres axillaris (Günther, 1864). Black axillary mojarra/mojarra malacapa. 2A, TR. UAS-0099. $\mathrm{N}=3$. Río Presidio en El Botadero. 18 mayo 2008.

Gerres cinereus (Walbaum, 1792).Yellowfin mojarra/ mojarra trompetera. 2A, AN. UAS-0094. N=2. Río Presidio en Las Garzas (desembocadura). 18 mayo 2008

Familia Haemulidae

Haemulon sexfasciatum Gill, 1862. Graybar grunt/ burro almejero. 2B, TR. UAS-0088. N=4. Río Presidio en El Botadero. 18 mayo 2008.

Familia Sciaenidae

Cynoscion squamipinnis (Günther, 1867). Scalyfin corvina/corvina aguada. 2B, TR. UAS-0171. $\mathrm{N}=2$. Río Presidio en El Botadero. 8 noviembre 2008.

Ophioscion scierus (Jordan \& Gilbert, 1884). Dusky croaker/corvineta parda. 2B, TR. UAS-0090. N=1. Río Presidio en El Botadero. 18 mayo 2008.

Familia Cichlidae

Cichlasoma beani (Jordan, 1889). Sinaloa cichlid/ mojarra de Sinaloa. 2C, NT. UABC-2064. N= 3. Río Presidio en la confluencia con Arroyo Copales. 13 septiembre 2008.

Oreochromis aureus (Steindachner, 1864). Blue Tilapia/tilapia azul. 2C, EX. UABC-2066. N= 1. Río Presidio en la confluencia con Arroyo Copales. 13 septiembre 2008.

\section{Familia Eleotridae}

Dormitator latifrons (Richardson, 1844). Pacific fat sleeper/puyeki. 1B, TR. UABC-2059. N=3. Río Presidio en El Botadero. 14 septiembre 2008.

Eleotris picta Kner, 1863. Spotted sleeper/guavina machada. 1B, TR. UABC-2063. N=1. Río Presidio en El Recodo. 14 septiembre 2008.

Gobiomorus maculatus (Günther, 1859). Pacific sleeper/dormilón manchado. 1B, TR. UABC-2069. $\mathrm{N}=1$. Río Presidio en Derivadora Siqueros, debajo de la cortina. 14 septiembre 2008.

\section{Familia Gobiidae}

Awaous banana (Valenciennes, 1837). River goby/ gobio de río. 1B, TR. UABC-2071. N=3. Río Presidio en El Recodo. 14 septiembre 2008.

Gobionellus microdon (Gilbert, 1892). Palmtail goby/ gobio cola de palma, 2A, TR. UABC-2054. $\mathrm{N}=3$. Río Presidio en El Botadero. 14 septiembre 2008.

Sicydium multipunctatum Regan, 1906. Multispotted goby/dormilón pecoso. 1B, TR. UABC-2083. N= 1. Río Presidio en Las Iguanas. 13 septiembre 2008.

\section{Familia Paralichthyidae}

Citharichthys gilberti Jenkins \& Evermann, 1889. Bigmouth sanddab/lenguado tapadera. 2A, TR. UAS-0024. N=1. Río Presidio en Las Garzas. 20 abril 2008 .

\section{Familia Achiridae}

Achirus mazatlanus (Steindachner, 1869). Pacific lined sole/tepalcate. 2A, TR. UABC 2060. $\mathrm{N}=1$. Río Presidio en El Botadero. 14 septiembre 2008.

Clasificación ecogeográfica: 1B Habitante permanente del conjunto estuarino-lagunar, 2A Especie del componente marino eurihalino, 2B Especie del componente marino estenohalino, 3A Especie catádroma, VI Especie vicaria, 1C Especie dulceacuícola primaria, y 2C Especie dulceacuícola secundaria. Clasificación zoogeográfica: NT Neotropical, NA Neártica, TR Tropical, AN Anfiamericana, AA Anfiatlántica, CT Circumtropical y EX Exótica.

con valores entre 25 (abril) y 33 (mayo), lo cual contrasta con la menor riqueza de 22 y 23 especies registrada en los meses de lluvia de agosto y septiembre, respectivamente (Cuadro 4).

Las especies con mayor abundancia relativa fueron Lile stolifera (10.6\%), Oreochromis aureus (9.2\%), A. banana (9.0\%), Anchoa analis (7.7 \%), Gobiomorus maculatus (6.4 $\%)$ y Atherinella crystallina (5.9\%), las cuales conjuntan el $48.8 \%$ del total de los ejemplares capturados durante el estudio (Cuadro 3). La abundancia en la captura por evento de muestreo fue mayor en febrero y mayo con $1177 \mathrm{y}$ 1174 individuos, respectivamente (Cuadro 4); sitios como El Botadero, Las Iguanas, BarrónOstial y El Recodo contribuyeron con el $65 \%$ de la captura total.

El cíclido exótico $O$. aureus, mostró amplia presencia a través del gradiente altitudinal en el río (2 a $150 \mathrm{msnm}$ ), mientras que otras especies como $A$. banana se registró en nueve sitios con altitudes entre 5 y 150 msnm (Cuadro 3). Otras especies de amplia distribución fueron $A$. crystallina, A. monticola y P. butleri que concurrieron en siete sitios entre los 4 y $150 \mathrm{msnm}$. En lo que respecta a especies restringidas a la parte media del río, destacan I. cf. pricei, P. latidens, 
CUADRO 3

Abundancia de especies ícticas por localidad de muestreo en las partes media (LH a ER) y baja (DS a LG) del Río Presidio, Mazatlán, Sinaloa; abril 2008-febrero 2009

TABLE 3

Abundance of fish species per sampling location in the middle (LH to ER) and lower (DS to LG) parts of Rio Presidio, Mazatlán, Sinaloa, during April 2008 to February 2009

\begin{tabular}{|c|c|c|c|c|c|c|c|c|c|c|c|c|}
\hline Taxa & LH & $\mathrm{LC}$ & LI & $\mathrm{LCO}$ & ER & DS & EW & $\mathrm{BO}$ & EB & LG & Total & $\% \mathrm{~N}$ \\
\hline Anchoa analis & 0 & 0 & 0 & 0 & 0 & 0 & 0 & 0 & 206 & 108 & 314 & 7.7 \\
\hline Lile stolifera & 0 & 0 & 0 & 0 & 0 & 0 & 5 & 0 & 244 & 184 & 433 & 10.6 \\
\hline Dorosoma smithi & 0 & 0 & 0 & 0 & 0 & 37 & 0 & 0 & 0 & 0 & 37 & 0.9 \\
\hline Ictalurus cf. pricei & 1 & 25 & 2 & 2 & 1 & 0 & 0 & 0 & 0 & 0 & 31 & 0.7 \\
\hline Ariopsis guatemalensis & 0 & 0 & 0 & 0 & 0 & 1 & 11 & 0 & 31 & 12 & 55 & 1.3 \\
\hline Ariopsis seemani & 0 & 0 & 0 & 0 & 0 & 0 & 0 & 0 & 2 & 2 & 4 & 0.1 \\
\hline Mugil cephalus & 0 & 0 & 0 & 0 & 0 & 0 & 5 & 87 & 63 & 10 & 165 & 4.0 \\
\hline Mugil curema & 0 & 0 & 0 & 0 & 0 & 0 & 0 & 10 & 10 & 8 & 28 & 0.7 \\
\hline Agonostomus monticola & 16 & 10 & 36 & 20 & 85 & 20 & 15 & 0 & 0 & 0 & 202 & 4.9 \\
\hline Atherinella crystallina & 38 & 38 & 35 & 8 & 54 & 44 & 0 & 0 & 23 & 0 & 240 & 5.9 \\
\hline Hyporhamphus rosae & 0 & 0 & 0 & 0 & 0 & 0 & 0 & 0 & 4 & 0 & 4 & 0.1 \\
\hline Poecilia butleri & 10 & 99 & 17 & 12 & 36 & 16 & 0 & 0 & 11 & 0 & 201 & 4.9 \\
\hline Poeciliopsis latidens & 30 & 140 & 5 & 1 & 21 & 2 & 0 & 0 & 0 & 0 & 199 & 4.9 \\
\hline Poeciliopsis presidionis & 0 & 77 & 35 & 2 & 42 & 7 & 0 & 0 & 0 & 0 & 163 & 4.0 \\
\hline Poeciliopsis prolifica & 0 & 0 & 1 & 4 & 0 & 0 & 0 & 0 & 0 & 0 & 5 & 0.1 \\
\hline Centropomus robalito & 0 & 0 & 0 & 0 & 0 & 0 & 0 & 0 & 11 & 7 & 18 & 0.4 \\
\hline Centropomus nigrescens & 0 & 0 & 0 & 0 & 0 & 1 & 8 & 0 & 9 & 1 & 19 & 0.5 \\
\hline Caranx vinctus & 0 & 0 & 0 & 0 & 0 & 0 & 0 & 0 & 1 & 0 & 1 & 0.02 \\
\hline Selene brevoorti & 0 & 0 & 0 & 0 & 0 & 0 & 0 & 0 & 3 & 3 & 6 & 0.2 \\
\hline Lutjanus novemfasciatus & 0 & 0 & 0 & 0 & 0 & 0 & 0 & 0 & 1 & 0 & 1 & 0.02 \\
\hline Lutjanus colorado & 0 & 0 & 0 & 0 & 0 & 0 & 0 & 0 & 4 & 2 & 6 & 0.2 \\
\hline Lutjanus argentiventris & 0 & 0 & 0 & 0 & 0 & 0 & 0 & 0 & 2 & 2 & 4 & 0.1 \\
\hline Diapterus brevirostris & 0 & 0 & 0 & 0 & 0 & 0 & 0 & 0 & 30 & 6 & 36 & 0.9 \\
\hline Eucinostomus entomelas & 0 & 0 & 0 & 0 & 0 & 0 & 0 & 0 & 4 & 0 & 4 & 0.1 \\
\hline Eucinostomus currani & 0 & 0 & 0 & 0 & 0 & 0 & 0 & 0 & 16 & 2 & 18 & 0.4 \\
\hline Eugerres axillaris & 0 & 0 & 0 & 0 & 0 & 0 & 0 & 0 & 150 & 31 & 181 & 4.4 \\
\hline Gerres cinereus & 0 & 0 & 0 & 0 & 0 & 0 & 0 & 0 & 53 & 28 & 81 & 2.0 \\
\hline Haemulon sexfasciatum & 0 & 0 & 0 & 0 & 0 & 0 & 0 & 0 & 5 & 1 & 6 & 0.2 \\
\hline Cynoscion squamipinnis & 0 & 0 & 0 & 0 & 0 & 0 & 0 & 0 & 2 & 0 & 2 & 0.1 \\
\hline Ophioscion scierus & 0 & 0 & 0 & 0 & 0 & 0 & 0 & 0 & 3 & 0 & 3 & 0.1 \\
\hline Cichasoma beani & 9 & 52 & 31 & 15 & 34 & 17 & 3 & 0 & 0 & 0 & 161 & 3.9 \\
\hline Oreochromis aureus & 16 & 72 & 70 & 22 & 134 & 38 & 3 & 11 & 7 & 4 & 377 & 9.2 \\
\hline Dormitator latifrons & 0 & 0 & 0 & 0 & 0 & 34 & 14 & 104 & 50 & 0 & 202 & 4.9 \\
\hline Eleotris picta & 0 & 0 & 1 & 9 & 3 & 4 & 0 & 120 & 0 & 1 & 138 & 3.4 \\
\hline Gobiomorus maculatus & 0 & 0 & 0 & 16 & 15 & 70 & 20 & 113 & 27 & 0 & 261 & 6.4 \\
\hline Awaous banana & 29 & 58 & 51 & 11 & 65 & 13 & 16 & 125 & 2 & 0 & 370 & 9.0 \\
\hline Gobionellus microdon & 0 & 0 & 0 & 0 & 0 & 0 & 0 & 2 & 2 & 1 & 5 & 0.1 \\
\hline Sicydium multipunctatum & 2 & 17 & 17 & 0 & 2 & 0 & 0 & 4 & 0 & 0 & 42 & 1.0 \\
\hline Citharichthys gilberti & 0 & 0 & 0 & 0 & 0 & 0 & 5 & 0 & 3 & 3 & 11 & 0.3 \\
\hline Achirus mazatlanus & 0 & 0 & 0 & 0 & 0 & 14 & 4 & 6 & 26 & 17 & 67 & 1.6 \\
\hline Total & 151 & 588 & 301 & 122 & 492 & 318 & 109 & 582 & 1005 & 433 & 4101 & \\
\hline$\% \mathrm{~N}$ & 3.68 & 14.34 & 7.34 & 2.97 & 12 & 7.75 & 2.66 & 14.2 & 24.5 & 10.6 & & \\
\hline Número de especies & 9 & 10 & 12 & 12 & 12 & 15 & 12 & 10 & 31 & 21 & 40 & \\
\hline
\end{tabular}

$\mathrm{LH}=$ Los Humayes, $\mathrm{LC}=$ Los Copales, $\mathrm{LI}=$ Las Iguanas, $\mathrm{LCO}=\mathrm{La}$ Cortina, $\mathrm{ER}=\mathrm{E} 1$ Recodo, $\mathrm{DS}=$ Derivadora Siqueros, $\mathrm{EW}=\mathrm{El}$ Walamo, $\mathrm{BO}=$ El Barrón-Ostial, $\mathrm{EB}=$ El Botadero y LG $=$ Las Garzas.

$\mathrm{LH}=$ Los Humayes, $\mathrm{LC}=$ Los Copales, $\mathrm{LI}=$ Las Iguanas, $\mathrm{LCO}=\mathrm{La}$ Cortina, $\mathrm{ER}=\mathrm{El}$ Recodo, DS $=$ Derivadora Siqueros, $\mathrm{EW}=\mathrm{E} 1$ Walamo, $\mathrm{BO}=\mathrm{E}$ l Barrón-Ostial, $\mathrm{EB}=\mathrm{E}$ l Botadero and $\mathrm{LG}=$ Las Garzas. 
CUADRO 4

Abundancia mensual de peces en la parte media y baja del Río Presidio, Mazatlán, Sinaloa; abril 2008-febrero 2009

TABLE 4

Monthly abundance of fish species in the middle and lower Río Presidio, Mazatlán, Sinaloa, during April 2008 to February 2009

\begin{tabular}{|c|c|c|c|c|c|c|c|c|}
\hline Especie & Abr.* & May.* & Ago.+ & Sep.+ & Nov.* & Feb.* & Total & $(\% \mathrm{~N})$ \\
\hline Anchoa analis & 62 & 224 & 15 & 5 & 0 & 8 & 314 & 7.7 \\
\hline Lile stolifera & 60 & 214 & 90 & 0 & 25 & 44 & 433 & 10.6 \\
\hline Dorosoma smithi & 10 & 2 & 10 & 15 & 0 & 0 & 37 & 0,9 \\
\hline Ictalurus cf. pricei & 0 & 0 & 3 & 2 & 25 & 1 & 31 & 0,8 \\
\hline Ariopsis guatemalensis & 7 & 2 & 7 & 3 & 28 & 8 & 55 & 1,3 \\
\hline Ariopsis seemanni & 0 & 2 & 0 & 2 & 0 & 0 & 4 & 0,1 \\
\hline Mugil cephalus & 9 & 18 & 10 & 17 & 40 & 71 & 165 & 4,0 \\
\hline Mugil curema & 0 & 0 & 0 & 10 & 6 & 12 & 28 & 0,7 \\
\hline Agonostomus monticola & 18 & 38 & 2 & 7 & 22 & 115 & 202 & 4,9 \\
\hline Atherinella crystallina & 89 & 62 & 28 & 12 & 10 & 39 & 240 & 5,9 \\
\hline Hyporhamphus rosae & 0 & 0 & 0 & 0 & 2 & 2 & 4 & 0,1 \\
\hline Poecilia butleri & 21 & 52 & 2 & 42 & 46 & 38 & 201 & 4,9 \\
\hline Poeciliopsis latidens & 0 & 13 & 0 & 0 & 21 & 165 & 199 & 4,9 \\
\hline Poeciliopsis presidionis & 0 & 43 & 21 & 30 & 14 & 55 & 163 & 4,0 \\
\hline Poeciliopsis prolifica & 4 & 1 & 0 & 0 & 0 & 0 & 5 & 0,1 \\
\hline Centropomus robalito & 6 & 8 & 0 & 2 & 1 & 1 & 18 & 0,4 \\
\hline Centropomus nigrescens & 1 & 1 & 0 & 4 & 13 & 0 & 19 & 0,5 \\
\hline Caranx vinctus & 0 & 0 & 1 & 0 & 0 & 0 & 1 & 0,02 \\
\hline Selene brevoorti & 6 & 0 & 0 & 0 & 0 & 0 & 6 & 0,2 \\
\hline Lutjanus novemfasciatus & 0 & 1 & 0 & 0 & 0 & 0 & 1 & 0,02 \\
\hline Lutjanus colorado & 2 & 2 & 0 & 0 & 1 & 1 & 6 & 0,2 \\
\hline Lutjanus argentiventris & 0 & 1 & 1 & 1 & 0 & 1 & 4 & 0,1 \\
\hline Diapterus brevirostris & 0 & 6 & 30 & 0 & 0 & 0 & 36 & 0,9 \\
\hline Eucinostomus currani & 1 & 0 & 0 & 0 & 17 & 0 & 18 & 0,4 \\
\hline Eucinostomus entomelas & 0 & 4 & 0 & 0 & 0 & 0 & 4 & 0,1 \\
\hline Eugerres axillaris & 6 & 132 & 0 & 0 & 35 & 8 & 181 & 4,4 \\
\hline Gerres cinereus & 0 & 20 & 0 & 0 & 50 & 11 & 81 & 2,0 \\
\hline Haemulon sexfasciatum & 0 & 5 & 0 & 0 & 0 & 1 & 6 & 0,2 \\
\hline Cynoscion squamipinnis & 0 & 0 & 0 & 0 & 2 & 0 & 2 & 0.5 \\
\hline Ophioscion scierus & 0 & 3 & 0 & 0 & 0 & 0 & 3 & 0,1 \\
\hline Cichlasoma beani & 38 & 35 & 11 & 19 & 31 & 27 & 161 & 3,9 \\
\hline Oreochromis aureus & 70 & 121 & 47 & 34 & 66 & 39 & 377 & 9.2 \\
\hline Dormitator latifrons & 10 & 10 & 15 & 50 & 14 & 103 & 202 & 4,9 \\
\hline Eleotris picta & 3 & 2 & 2 & 1 & 5 & 125 & 138 & 3,4 \\
\hline Gobiomorus maculatus & 17 & 64 & 17 & 8 & 20 & 135 & 261 & 6,4 \\
\hline Awaous banana & 65 & 52 & 87 & 15 & 6 & 145 & 370 & 9,0 \\
\hline Gobionellus microdon & 1 & 1 & 1 & 2 & 0 & 0 & 5 & 0,1 \\
\hline Sicydium multipunctatum & 4 & 15 & 0 & 2 & 9 & 12 & 42 & 1,0 \\
\hline Citharichthys gilberti & 1 & 2 & 1 & 0 & 2 & 5 & 11 & 0,3 \\
\hline Achirus mazatlanus & 11 & 21 & 4 & 1 & 28 & 2 & 67 & 1,6 \\
\hline Total & 522 & 1177 & 405 & 284 & 539 & 1174 & 4101 & \\
\hline$\% \mathrm{~N}$ & 12.72 & 28.70 & 9.88 & 6.93 & 13.14 & 28.62 & & \\
\hline Número de especies & 25 & 33 & 22 & 23 & 27 & 27 & 40 & \\
\hline
\end{tabular}

Meses de lluvia $(+)$ y de secas $(*)$. Rainy $(+)$ and dry $(*)$ months. 
P. presidionis y $P$. prolifica, en altitudes entre 33 y 150 msnm (Cuadro 3).

En relación con la similitud de especies entre sitios de muestreo, los resultados mostraron una menor distancia $(<15 \%)$ en localidades de la parte media de la cuenca, especialmente entre La Cortina y Los Humayes, así como entre Las Iguanas, Los Copales y El Recodo (16\%) (Fig. 2). Por periodo de muestreo y a un nivel de corte del $45 \%$ de disimilitud, dos grupos son formados; el primero por los meses de agosto y septiembre 2008 que corresponden al periodo de lluvias, y el segundo grupo integrado por dos subgrupos (abril y mayo 2008, agosto 2008 y febrero 2009) que corresponden al periodo de secas (Fig. 3). Los meses de agosto 2008 y febrero 2009 registraron una menor distancia de enlace (24\%).

En el análisis de componentes principales (Fig. 4) indica que el primer componente explicó el $58.3 \%$ de la variación, aportando una mayor contribución las variables de riqueza de especies $(0.220)$ y salinidad $(0.215)$ (Cuadro
5). El segundo componente explicó el $21.4 \%$ de la variación, donde las variables con mayor contribución fueron la temperatura (0.299) y abundancia total de las especies (0.273).

La riqueza de especies y la salinidad mostraron un mayor grado de asociación (Fig. 4), mostrando un mayor número de especies en aquellas localidades de la parte baja del río donde la salinidad se incrementa por la influencia de flujos de marea y favorece la mayor incursión de especies de estirpe marina o "periféricas". De igual manera, ambas salinidad y riqueza de especies tuvieron una relación inversa con la altitud. Por su parte, la temperatura y la abundancia total de las especies mostraron una relación directa (Fig. 4).

Derivación ecológica y consideraciones zoogeográficas: De acuerdo a los resultados, respecto a la derivación ecológica de las especies registradas para el Río Presidio, la mayoría de los taxa corresponden al componente marino-eurihalino $(17$ especies $=43 \%$; Cuadro 2$)$

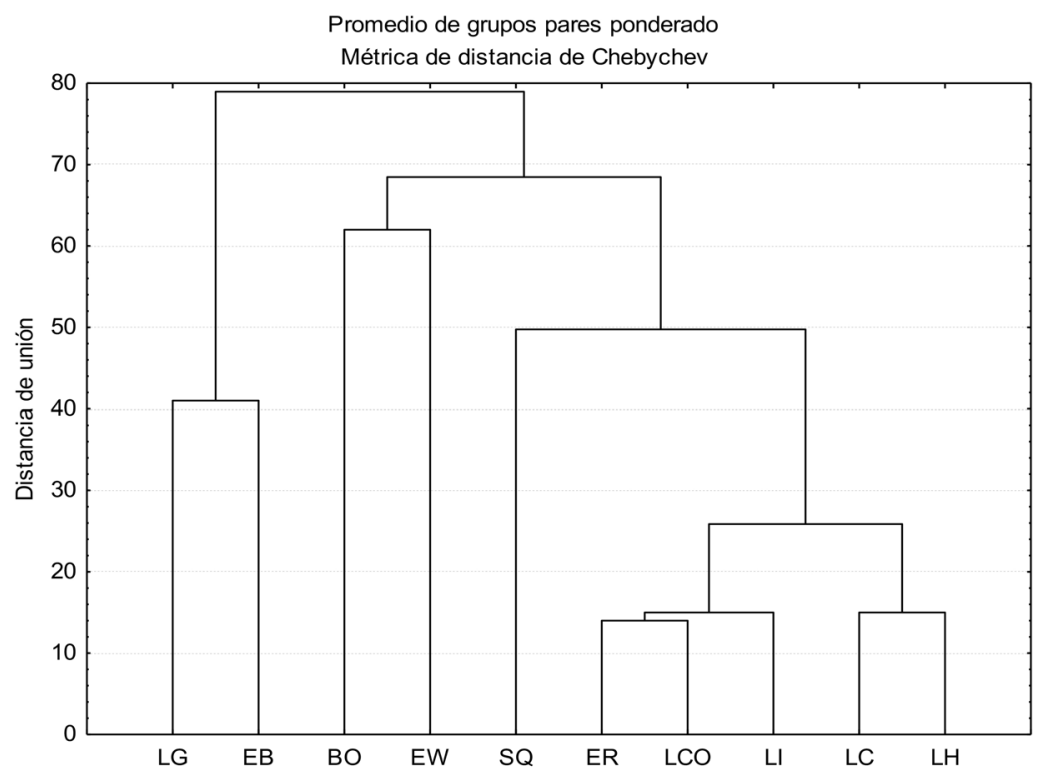

Fig. 2. Disimilitud de especies ícticas por localidad de muestreo en el Río Presidio, Mazatlán, Sinaloa, de abril 2008 a febrero 2009. $\mathrm{LH}=$ Los Humayes, $\mathrm{LC}=$ Los Copales, $\mathrm{LI}=$ Las Iguanas, LCO $=$ La Cortina, ER $=\mathrm{El}$ Recodo, DS $=$ Derivadora Siqueros, EW = El Walamo, $\mathrm{BO}=$ El Barrón-Ostial, EB = El Botadero y LG = Las Garzas.

Fig. 2. Dissimilarity of fish species by sampling site in the Rio Presidio, Mazatlán, Sinaloa, during April 2008 to February 2009. $\mathrm{LH}=$ Los Humayes, LC $=$ Los Copales, LI = Las Iguanas, LCO = La Cortina, ER = El Recodo, DS = Derivadora Siqueros, $\mathrm{EW}=\mathrm{El}$ Walamo, $\mathrm{BO}=\mathrm{El}$ Barrón-Ostial, $\mathrm{EB}=\mathrm{El}$ Botadero and $\mathrm{LG}=\mathrm{Las}$ Garzas. 


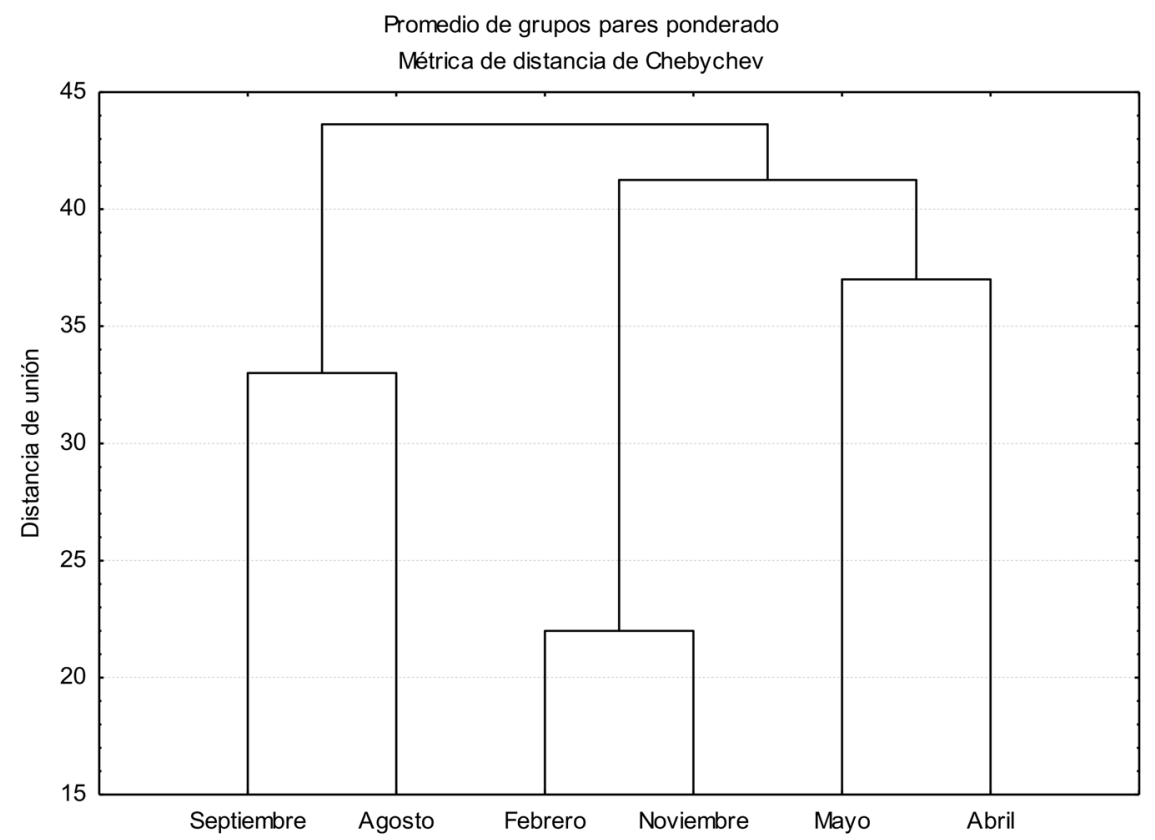

Fig. 3. Similitud de especies ícticas por mes de muestreo en la parte baja y media del Río Presidio, Mazatlán, Sinaloa, de abril 2008 a febrero 2009.

Fig. 3. Similarity of fish species by month of sampling in the lower and middle Río Presidio, Mazatlán, Sinaloa, during April 2008 to February 2009.

\section{CUADRO 5}

Contribución de variables basadas en correlaciones sobre los atributos de altitud y de riqueza de especies ícticas, en la parte baja y media del Río Presidio, Sinaloa, México. Los dos valores más significativos en cada factor se indican en negrillas

TABLE 5

Contribution of variables based on correlations in function of altitude and fish species richness in the middle and lower parts of Río Presidio, Sinaloa, Mexico. The two most significant values for each factor are depicted in bold

\begin{tabular}{lccccc} 
& Factor 1 & Factor 2 & Factor 3 & Factor 4 & Factor 5 \\
Oxígeno & 0.141796 & 0.198335 & $\mathbf{0 . 0 9 7 3 4 0}$ & 0.020820 & $\mathbf{0 . 2 3 9 2 4 5}$ \\
pH & 0.163199 & 0.186844 & 0.038498 & 0.015649 & 0.078258 \\
Temperatura & 0.057787 & $\mathbf{0 . 2 9 9 1 2 4}$ & 0.000351 & $\mathbf{0 . 6 3 0 4 1 1}$ & 0.001746 \\
Salinidad & $\mathbf{0 . 2 2 0 1 1 5}$ & 0.006372 & 0.065040 & 0.000446 & 0.131279 \\
Turbidez & 0.104062 & 0.033570 & $\mathbf{0 . 6 9 2 3 7 4}$ & 0.029731 & 0.066792 \\
Riqueza de Especies & $\mathbf{0 . 2 1 4 8 4 1}$ & 0.003037 & 0.074414 & 0.018521 & $\mathbf{0 . 3 5 9 3 9 2}$ \\
Abundancia Total & 0.098201 & $\mathbf{0 . 2 7 2 7 1 8}$ & 0.031983 & $\mathbf{0 . 2 8 4 4 2 1}$ & 0.123289 \\
\hline
\end{tabular}

En menor proporción estuvieron representadas las especies del componente marino estenohalino con siete taxa (18\%), seguidas por elementos dulceacuícolas secundarios y elementos permanentes del conjunto estuarino-lagunar, ambos con seis especies (15\%) cada uno. Por su parte, los elementos vicarios estuvieron representados solamente por dos especies (5 $\%$, A. crystallina y D. smithi), el componente dulcecacuícola primario por Ictalurus $\mathrm{cf}$. pricei ( $2 \%$ ) y, finalmente, el catádromo por $A$. monticola (2\%; Cuadro 2). 


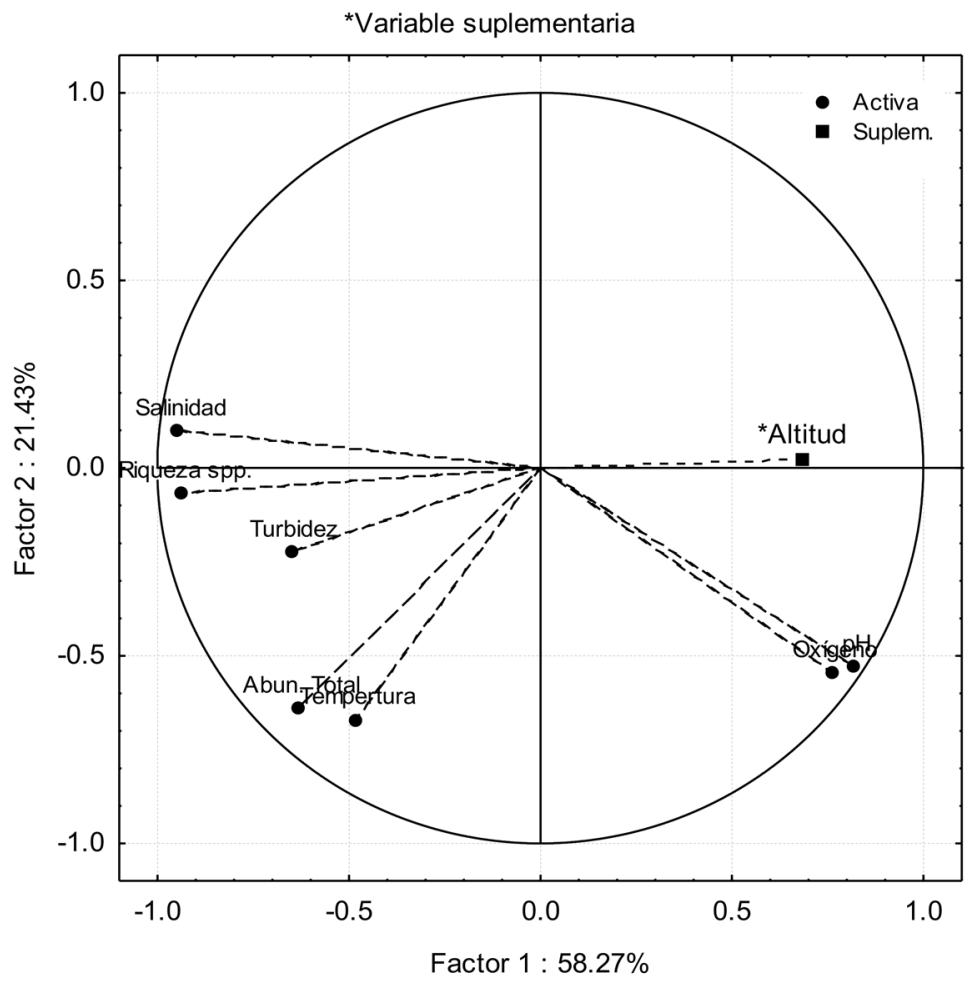

Fig. 4. Proyección de las variables activas y la variable suplementaria (altitud) sobre el plano factorial 1 y 2 , derivado del análisis de componentes principales para la ictiofauna de la parte media y baja del Río Presidio, Sinaloa, México (de abril 2008 a febrero 2009).

Fig. 4. Projection of the active variables and supplementary variable (altitude) on the factor-plane (1x2), derived from principal component analysis for the fish fauna in the middle and lower Río Presidio, Sinaloa, Mexico (April 2008 to February 2009).

Zoogeográficamente, seis especies del conjunto neotropical están representadas en la cuenca media del Río Presidio (A. crystallina, P. butleri, P. latidens, P. presidionis, $P$. prolifica y $C$. beani); mientras que las dos especies vicarias en la parte medida de esta cuenca (D. smithi e I. cf. pricei) son de afinidad neártica. Las 31 especies restantes estuvieron representadas por elementos de derivación marino-eurihalina o periférica, de las cuales 27 son afines a la región del Pacífico oriental tropical (Cuadro 2). En esta última región se distribuyen 18 especies en las provincias SinusCaliforniana, Mexicana y Panámica (Cuadro 2), de las cuales siete extienden su distribución hacia la provincia Sandieguina (Caranx vinctus, Lutjanus argentiventris, L. colorado, L. novemfasciatus, E. currani, E. picta y $A$. banana). Otras especies como Hyporhamphus rosae, habitan dentro de las provincias Sandieguina, Sinus-californiana y Mexicana; mientras que $A$. analis, su distribución abarca las provincias Sinus-californiana y Mexicana, pero no alcanza a la provincia Panámica.

Por otro lado, especies como A. monticola y $G$. cinereus representaron elementos anfiamericanos; en tanto que $M$. cephalus y M. curema, constituyeron los únicos elementos circumtropicales y anfiatlánticos, respectivamente. La única especie exótica, $O$. aureus, es de afinidad etiópica.

\section{DISCUSIÓN}

A través del estudio ictiológico de la cuenca media y baja del Río Presidio, se registró 
un total de 40 especies, cifra que constituye el $56 \%$ de las especies ícticas reportadas para las aguas continentales del Estado de Sinaloa (Jordan, 1895; Álvarez del Villar 1970; CastroAguirre et al., 1999; Miller et al., 2005).

Es importante destacar que la mayoría de las especies registradas $(\mathrm{n}=39)$ son nativas del Río Presidio (sensu Miller et al., 2005), donde sobresalen endemismos de tipo regional o compartido para el Estado de Sinaloa: $D$. smithi, A. crystallina, P. latidens, $P$. presidionis, $P$. prolifica y $C$. beani (Van der Heiden \& Plascencia-González, 2002). Una de éstas, Poeciliopsis latidens es considerada de acuerdo a la Norma Oficial Mexicana [NOM-059-2010] (DOF 2010), como una especie amenazada.

Sólo una especie exótica fue registrada, el cíclido $O$. aureus, que presentó alta abundancia y amplia distribución en el área de estudio, y que muestra un patrón similar en otras cuencas hidrológicas del Estado de Sinaloa (Ruiz-Campos et al., 2014).

En términos de la abundancia (numérica) de las especies, sobresalió la presencia de aquellas que exhibieron un comportamiento gregario al momento de su captura: L. stolifera, $O$. aureus, A. banana, A. analis, G. maculatus y $A$. crystallina. La mayor abundancia mensual de organismos en de febrero y mayo, podría ser atribuida a la disminución de nivel y flujo del río durante el periodo de estiaje, situación que favorece el hacinamiento de los peces y facilita de este modo su captura. Las familias con mayor riqueza de especies fueron aquellas características de los sitios costeros con ambientes de tipo oligohalino e hiperhalino o eurihalino como El Botadero y Las Garzas (32 especies de manera combinada), que favorecen la incursión de especies de estirpe marina (eurihalinas y estenohalinas), en respuesta a la elevada producción primaria y heterogeneidad ambiental de estos ecosistemas (YáñezArancibia et al., 1980; González-Acosta et al., 2017); así como por el uso que hacen de estas áreas como zonas de crianza y reclutamiento (González-Acosta et al., 2015).

Con base en la clasificación ecogeográfica de las especies que habitan las lagunas costeras y sistemas fluvio-estuarinos del Estado de Sinaloa, Castro-Aguirre et al. (1999), reportaron la existencia de 226 especies, de las cuales 107 están presentes en la región sur. Esta riqueza íctica se apoya en estudios de exploración concentrados únicamente en la laguna Huizache-Caimanero y estuario de Mazatlán (Castro-Aguirre et al. 1999), los cuales tienen flujo permanente de agua y una mayor superficie en comparación con la zona de estuario del Río Presidio, cuya desembocadura es obstruida durante el periodo de estiaje por una barra arenosa que impide el flujo de agua y con ello la dispersión de muchas especies que incursionan temporalmente o de manera esporádica a los ambientes estuarinos. Dicha situación provocó una reducción en el número de especies registradas (un total de 32) en la parte baja de la cuenca del río; de las cuales, 23 son de interés comercial (Fischer et al., 1995) y son aprovechadas por la población pesquera local.

Por su parte, la riqueza de especies fue mayor en la zona baja de la cuenca, en las localidades de El Botadero y Las Garzas, en las cuales se registró la presencia de especies de derivación marino eurihalina o periféricas procedentes del litoral costero. La mayor riqueza de especies en sitios próximos a la desembocadura al mar es un patrón característico en los sistemas fluvio-estuarinos del Pacífico mexicano (Castro-Aguirre et al., 1999; GonzálezAcosta et al., 2017).

A nivel temporal, la composición íctica en el área de estudio fue influenciada por las condiciones hidrológicas que fueron contrastantes en el ecosistema, donde el periodo de lluvias corresponde a agosto y septiembre y el resto de los meses al período de secas o estiaje. La incursión de especies marino eurihalinas a la parte baja del río se incrementó durante el estiaje, como frecuentemente ocurre en muchos ecosistemas fluvio-estuarinos de México (Castro-Aguirre et al., 1999).

En relación a la clasificación ecogeográfica de las especies según su tolerancia a la salinidad y su distribución zonal a través del río, en este estudio se incluyeron dos tipos de ecosistemas (dulceacuícola y marino 
oligohalino-polihalino); esto se reflejó en los resultados, ya que los peces presentes en la parte media del Río Presidio, están representados en su mayoría por elementos dulceacuícolas del tipo secundario (seis especies), los cuales tienen una poca a moderada tolerancia a la salinidad (Myers, 1951; Miller, 1966; Miller et al., 2005).

La especie D. smithi, ubicada dentro del componente vicario por Castro-Aguirre et al. (1999), se encontró solo en un sitio (Derivadora Siqueros), mismo que le aportó condiciones necesarias para el completo desarrollo de esta especie, que por sus características morfológicas requiere de zonas de corriente y de mayor profundidad. Las especies de eleótridos $(D$. latifrons, E. picta y G. maculatus) se comportaron como invasores eurihalinos en el área de estudio, encontrándose en salinidades de 0.1 a $23 \%$. Estas mismas especies, junto con el gobio A. banana forman parte del componente complementario en los ambientes dulceacuícolas de la Península de Baja California [cf. A. tajasica en Ruiz-Campos et al. (1999) y RuizCampos et al. (2003)]. Dentro de la cuenca del Río Presidio, el componente dulceacuícola secundario estuvo representado por especies de las familias Cichlidae y Poeciliidae, mientras que el componente primario por la familia Ictaluridae. En este último caso, la única especie aquí representada fue $I$. cf. pricei, misma que se encontró en los sitios de la parte media de la cuenca.

Con base en la distribución de especies a través de la parte media y baja del Río Presidio, fue notable una zonación que se representa de la siguiente manera, en la parte baja de este río (localidades de El Walamo, Barrón-Ostial, El Botadero, Las Garzas y Derivadora Siqueros) por especies marinas de tipo eurihalino; y en la parte media por especies dulceacuícolas como P. butleri, P. latidens, $P$. presidionis, $P$. prolifica y el exótico $O$. aureus.

La mayoría de las especies registradas en el área de estudio son de estirpe marina, las cuales poseen ámbitos de distribución que incluyen la Región del Pacífico oriental, específicamente dentro de las provincias
Sandieguina, Sinus-Californiana, Mexicana y Panámica (Briggs, 1974); aunque no todas esas especies se ubicaron de forma restringida en las cuatro provincias biogeográficas antes citadas, en todos los casos se distribuyen en al menos dos de éstas; por ejemplo, A. analis solo se distribuye dentro de las provincias Sinus-californiana y Mexicana (Van der Heiden \& Plascencia-González, 2002). Asimismo, las especies pertenecientes a las familias Poeciliidae, Atherinopsidae y Cichlidae, son derivadas de grupos de estirpe sudamericana, particularmente de la región amazónica, que históricamente se han dispersado hacia las regiones tropicales del continente (De la Vega-Salazar, 2003; Miller et al., 2005). Otras especies como A. monticola y G. cinereus se distribuyen tanto en la vertiente del Pacífico como del Golfo de México (Castro-Aguirre et al., 1999), mientras que M. cephalus y M. cure$m a$ son formas de distribución circumtropical y anfiatlántica, respectivamente.

Finalmente, se establece que el estudio sobre la distribución y abundancia espaciotemporal de la ictiofauna de la parte media y baja del Río Presidio, previo a la construcción de la Presa Picachos, servirá de base y referencia para evaluar los futuros impactos de esta obra hidráulica sobre las comunidades de peces nativos, especialmente del componente catádromo que utiliza estos biotopos como parte importante de su ciclo de vida.

\section{AGRADECIMIENTOS}

Las siguientes personas apoyaron los muestreos ictiológicos: Leonardo Ayala-Rodríguez, Leonardo Ayala Baldenegro, Daniel Montoya López, Igmar Sosa Cornejo, y Reynaldo Ayala Baldenegro. A Carlos Chon-López por la elaboración de la figura de área de estudio. Apoyo económico y logístico fue recibido a través de los cuerpos académicos de Biología Molecular y Vida Silvestre (Universidad Autónoma de Sinaloa), Estudios Relativos a la Biodiversidad (Universidad Autónoma de Baja California) y Biología de la Conservación (Universidad Autónoma de Nuevo León). 
GRC y MLLV agradecen el apoyo de SNICONACyT, y AFGA agradece también los apoyos de los programas COFAA y EDI-IPN, y SNI-CONACyT. Finalmente a tres revisores anónimos por los útiles comentarios y recomendaciones al manuscrito.

\section{RESUMEN}

El Río Presidio, ubicado en el sur de Sinaloa, México, pertenece a la zona de transición zoogeográfica NeárticoNeotropical, donde su fauna de peces podría manifestar cambios en la abundancia y distribución de las especies durante los períodos de lluvias y de secas. El objetivo principal de este estudio fue describir la composición taxonómica y la abundancia espacial y temporal de la fauna de peces de la parte baja y media del Río Presidio, mediante muestreos bimestrales de abril 2008 a febrero 2009. Cuarenta especies (39 nativas y 1 exótica) pertenecientes a 32 géneros y 19 familias fueron registradas, siendo las familias Gerreidae y Poeciliidae las más diversas con cinco y cuatro especies, respectivamente. Seis especies: Lile stolifera, Oreochromis aureus, Awaous banana, Gobiomorus maculatus, Anchoa analis y Atherinella crystallina, en ese orden de importancia contribuyeron con casi el $49 \%$ de la abundancia íctica total. La riqueza de especies y la salinidad demostraron una relación directa y ambas tuvieron una relación inversa con la altitud. Con base en la disimilitud de especies entre sitios, dos tipos de ensambles de peces fueron distinguidos a través de un gradiente de salinidad y turbidez en el río, el primero representado por especies de derivación marina (periféricas) en la parte baja, y el segundo por especies dulceacuícolas secundarias en la parte media. La mayor similitud de especies se dio en agosto y septiembre y estuvo asociada a condiciones de altos flujos del periodo de lluvias. Veintisiete especies tienen afinidad a la Región del Pacífico oriental, seis a la Región Neotropical, dos a la Región Neártica (Dorosoma smithi e Ictalurus cf. pricei), una Anfiatlántica (Mugil curema), una Circumtropical (M. cephalus), dos Anfiamericanas (Agonostomus monticola y Gerres cinereus) y una exótica (O. aureus).

Palabras clave: ictiofauna; distribución; abundancia; zoogeografía; ecogeografía.

\section{REFERENCIAS}

Álvarez del Villar, J. (1970). Peces mexicanos (claves). México, D.F.: Instituto Nacional de Investigaciones Biológico Pesqueras. Secretaría de Industria y Comercio.

Briggs, J. C. (1974). Marine zoogeography. Nueva York: McGraw-Hill.
Castro-Aguirre, J. L. (1976). Catálogo sistemático de los peces marinos que penetran a las aguas continentales de México, con aspectos zoogeográficos y ecológicos. Serie Científica 19. México, D.F.: Departamento de Pesca.

Castro-Aguirre, J. L., Espinosa-Pérez, H. S. \& SchmitterSoto, J. J. (1999). Ictiofauna estuarino-lagunar y vicaria de México. México, D.F.: Limusa.

Darlington, P. J. Jr. (1963). Zoogeography, the geographical distribution of animals. Nueva York: Wiley.

De la Lanza, G. \& García-Calderón, J. L. (1991). Sistema lagunar Huizache y Caimanero, Sin. Un estudio socio ambiental, pesquero y acuícola. Hidrobiológica, 1,15 .

De la Vega-Salazar, M. Y. (2003). Situación de los peces dulceacuícolas en México. Ciencias (UNAM), 72, 20-30.

Diario Oficial. (2010). Norma Oficial Mexicana NOM059-Ecol-2010, que determinan las especies y subespecies de flora y fauna silvestres terrestres y acuáticas en peligro de extinción amenazadas, raras y las sujetas a protección especial, y que establece especificaciones para su protección. 6 de marzo, México, D.F.

Espinosa-Pérez, H., Fuentes-Mata, P., Gaspar-Dillanes, M. T., \& Arenas, V. (1993). Notes on Mexican Ichthyofauna. In T. P. Ramamoorthy, R. Bye, A. Lot \& J. Fa (Eds.), Biological diversity of Mexico: origins and distribution (pp. 229-251). Nueva York: Oxford University Press.

Fischer, W., Krupp, F., Schneider, W., Sommer, C., Carpenter, K. E., \& Niem, V. H. (1995). Guía FAO para la identificación de especies para los fines de pesca, Pacifico Centro- Oriental. FAO: Roma.

García, E. (2004). Modificaciones al Sistema de Clasificación Climática de Köppen. Libro 6. México, D.F.: Instituto de Geografía-Universidad Nacional Autónoma de México.

González-Acosta, A. F., Béarez, P., Álvarez-Pliego, N., De La Cruz-Agüero, J., \& Castro-Aguirre, J. L. (2007). On the taxonomic status of the Diapterus peruvianus (Cuvier, 1830) and reinstatement of Diapterus brevirostris (Sauvage, 1879) (Teleostei: Gerridae). Cybium, 31, 369-377.

González-Acosta, A. F., Ruiz-Campos, G., \& Balart, E. F. (2015). Composition and zoogeography of fishes in mangrove ecosystems of Baja California Sur, México. En R. Riosmena-Rodríguez, A. F. González-Acosta, \& R. Múñiz-Salazar (Eds.), The arid mangroves from Baja California Peninsula (Vol 1, Chapter 5, pp. 63-80). Nueva York, USA: Nova Science Publishers, Inc.

González-Acosta, A. F., Rodiles-Hernández, R. \& González-Díaz, A. A. (2017). Checklist of the marine and 
estuarine fishes of Chiapas, Mexico. Marine Biodiversity, 2017: 1-16. DOI: 10.1007/s12526-016-0630-y.

INEGI. (1995). Estudio hidrológico del Estado de Sinaloa. México, D.F.: Gobierno del Estado de Sinaloa.

Jordan, D. S. (1895). The fishes of Sinaloa. Proceedings of the California Academy of Sciences 5, 377-514.

Miller, R. R. (1966). Geographical distribution of Central American freshwater fishes. Copeia, 4, 773-802.

Miller, R. R., Minckley, W. L., \& Norris, S. M. (2005). Freshwater fishes of Mexico. Chicago: Chicago University Press.

Myers, G. S. (1951). Fresh-water fishes and East Indian zoogeography. Stanford Ichthyological Bulletin, 4, $11-21$.

Oberdorff, T., Pont, D., Hugueny, B., \& Porcher, J. P. (2002). Development and validation of a fish-based index for the assessment of 'river health' in France. Freshwater Biolology, 47, 1720-1734.

Page, L. M., Espinosa-Pérez, H., Findley, L. T., Gilbert, C. R., Lea, R. N., Mandrak, N. E., Mayden, R. L., \& Nelson, J. S. (2013). Common and scientific names of fishes from the United States, Canada and Mexico. Maryland: American Fisheries Society, Special Publication 34.

Ruiz-Campos, G., Castro-Aguirre, J. L., GonzálezGuzmán, S., \& Sánchez-Gonzáles, S. (1999). First records of two tropical gobies, Awaous tajasica and Ctenogobius sagittula (Pisces: Gobiidae), in the continental waters of Baja California, México. Bulletin of the Southern California Academy of Sciences, 98,131-136.

Ruiz-Campos, G., Castro-Aguirre, J. L., Contreras-Balderas, S., Lozano-Vilano, M. L., González-Acosta, A. F., \& Sánchez-Gonzáles, S. (2003). An annotated distributional checklist of the freshwater fish from Baja California Sur, México. Reviews in Fish Biology and Fisheries, 12, 143-155.
Ruiz-Campos, G., Varela-Romero, A., Sánchez-Gonzáles, S., Camarena-Rosales, F., Maeda-Martínez, A. M., González-Acosta, A. F., Andreu-Soler, A., Campos-González, E., \& Delgadillo-Rodríguez, J. (2014). Peces invasores del noroeste de México. In R. E. Mendoza-Alfaro \& P. Kolef (Eds.), Especies Acuáticas Invasoras en México (pp. 375-399). México: CONABIO.

Sicairos-Avitia, S., Díaz, J. \& Sánchez-Gonzáles, S. (2003). Recursos bióticos de la zona costera de Sinaloa. In C. Karam-Quiñones, \&. J. L. Berau-Lozano (Eds.), Sinaloa y su Ambiente: Visiones del Presente y Perspectivas (pp. 281-328). Sinaloa: Universidad Autónoma de Sinaloa.

Tamayo, J. L. \& West, R. C. (1964). Handbook of Middle American Indians, J. Natural Environment and Early Cultures. In R.C. West (Ed.), The hydrography of Middle America (pp. 84-121). Austin: University of Texas Press.

Van der Heiden, A. M. \& Plascencia-González, H. G. (2002). La fauna endémica del Estado de Sinaloa. In J. L. Cifuentes-Lemus, \& J. Gaxiola-López (Eds.), Atlas de la biodiversidad de Sinaloa (pp. 423-429). Sinaloa: El Colegio de Sinaloa.

Vega-Aviña, R. (2002). Flora Vascular de Sinaloa. In J. L. Cifuentes-Lemus, \& J. Gaxiola-López (Eds.), Atlas de la Biodiversidad de Sinaloa (pp. 69-79). Sinaloa: El Colegio de Sinaloa.

Watson, R. E. (1996). Revision of the subgenus Awaous (Chonophorus) (Teleostei: Gobiidae). Ichthyolocal Exploration in Freshwater, 7, 1-18.

Yáñez-Arancibia, A., Amezcua-Linares, F., \& Day, J. W., Jr. (1980). Fish community structure and function in Términos Lagoon, a tropical estuary in southern Gulf of Mexico. In V. Kennedy (Ed.), Estuarine perspectives (pp. 465-482). The Fifth Biennial International Estuarine Research Federation Conference. Nueva York: Academic Press. 